10. Mulcahy JJ, Furlow WL. Vaginal metastasis from renal cell carcinoma: Radiographic evidence of possible route of spread. J Urol. 1970;104:50-2.

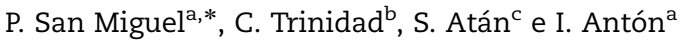

${ }^{a}$ Servicio de Anatomía Patológica, Hospital Povisa, Vigo,

Pontevedra, España
${ }^{\mathrm{b}}$ Servicio de Rayos, Hospital Povisa, Vigo, Pontevedra, España

'Servicio de Ginecología, Hospital Povisa, Vigo, Pontevedra, España

*Autor para correspondencia.

Correo electrónico: psanmiguel@povisa.es (P. San Miguel).

\title{
Graves consecuencias del tratamiento mediante esclerosis con ácido acético en un caso de linfangioma quístico retroperitoneal
}

\section{Serious consequences of the treatment by sclerotherapy with acetic acid in a case of retroperitoneal cystic lymphangioma}

\section{Sr. Director:}

Los tumores retroperitoneales constituyen un conjunto de neoplasias poco frecuentes derivadas de los vasos, los nervios, el tejido muscular y los restos embrionarios ${ }^{1}$. Entre los distintos tipos encontramos el linfangioma quístico retroperitoneal. Este es un tumor benigno y con escasa incidencia, cuyo origen radica en una alteración del desarrollo del sistema linfático².

Presentamos un caso de linfangioma quístico retroperitoneal tratado con ácido acético y con el resultado de necrosis de la pared del colon derecho y graves complicaciones posteriores.

Varón de 45 años con antecedentes personales de dislipemia, litiasis renal e intervenido hace 30 años de linfangioma quístico retroperitoneal a través de laparotomía (no aporta informes) que consulta en el servicio de digestivo por dolor en la zona lumbar derecha de tres meses de duración irradiado a flanco derecho las últimas semanas sin otra sintomatología acompañante. En la exploración se palpa una masa en el hemiabdomen derecho. Se procede así a realizar una tomografía axial computarizada (TAC) abdominopélvico (fig. 1), donde se evidencia una voluminosa masa quística polilobulada retroperitoneal en el hemiabdomen derecho compatible con linfangioma macroquístico retroperitoneal; la masa rodea y desplaza el riñón derecho, el colon ascendente, la cabeza del páncreas, la $2 .^{\mathrm{a}}$ y la $3 .^{\mathrm{a}}$ porción duodenal, la cava inferior, la aorta y los vasos ilíacos. Debido a la relación tan estrecha del linfangioma con estructuras vitales se rechaza la cirugía.

Se procede así a la inyección de ácido acético como tratamiento alternativo. Bajo control de la TAC se drena el quiste, obteniéndose $1.300 \mathrm{~cm}^{3}$ de líquido lechoso. Una vez descartadas las comunicaciones anómalas con el resto del retroperitoneo (al inyectar contraste yodado), se esclerosa con $100 \mathrm{~cm}^{3}$ de ácido acético al $40 \%$ y tras 20 min se extrae y se lava abundantemente con suero, dejando un drenaje.

Durante los días posteriores, el paciente presenta dolor abdominal controlado con analgesia y hematuria autolimitada las primeras $24 \mathrm{~h}$, que se atribuye a inflamación ureteral. Cuatro días después se realiza una TAC de control, en la cual se objetiva una esclerosis casi total de linfangioma (pequeño lóculo residual de $2 \times 1 \mathrm{~cm}$ ), por lo que se retira el drenaje.

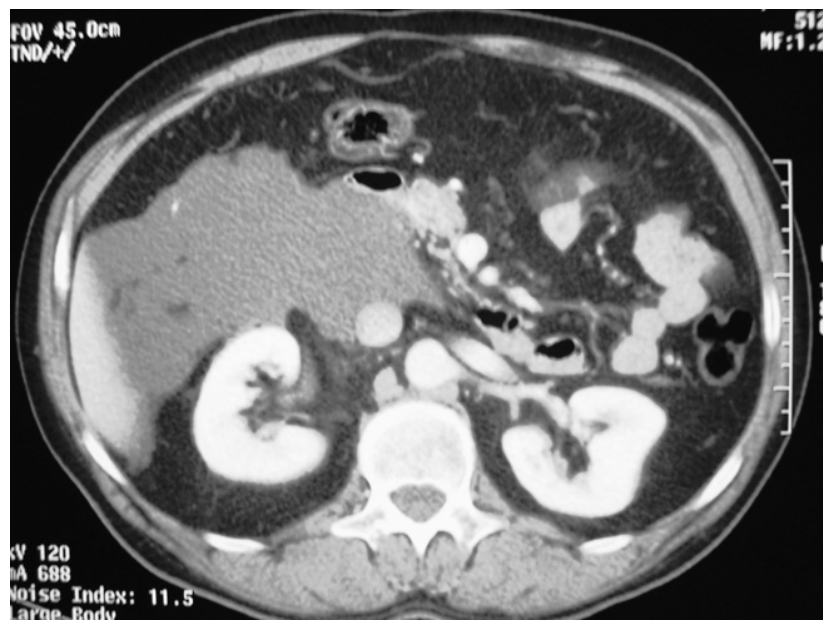

Figura 1 - Tomografía axial computarizada abdominopélvica con contraste oral e intravenoso. Se visualiza una voluminosa masa quística polilobulada retroperitoneal en el hemiabdomen derecho que rodea y desplaza el riñón derecho, el colon ascendente, la cabeza del páncreas, la $2 .^{a}$ y la 3. ${ }^{a}$ porción duodenal, la cava inferior, la aorta y los vasos ilíacos. 


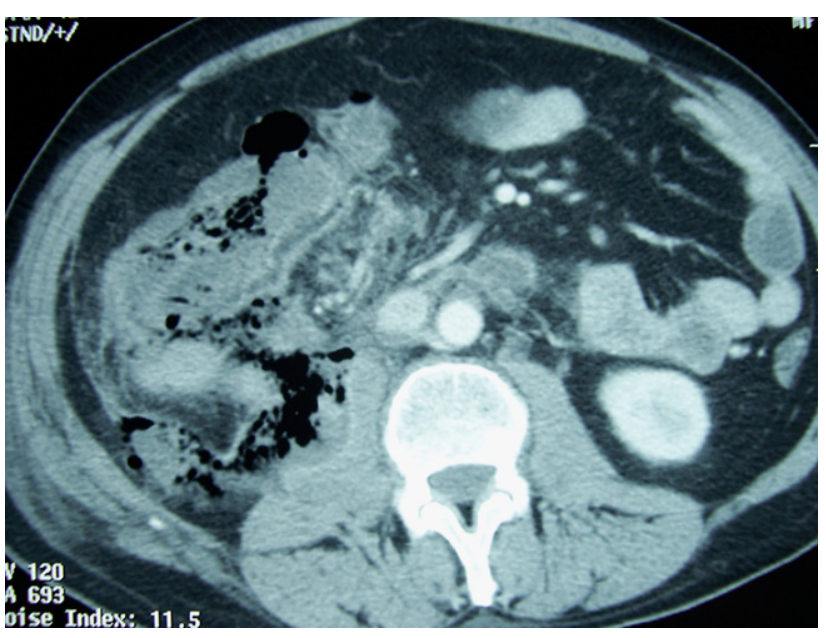

Figura 2 - Tomografía axial computarizada abdominal con contraste oral e intravenoso. Se aprecia una extensa colección retroperitoneal derecha con perforación del colon ascendente y otra pequeña periduodenal.

Durante los días siguientes comienza con dolor y parestesias en la zona inguinal derecha y con picos febriles. Se hace una nueva TAC de control, donde se aprecia una colección retroperitoneal anterior al psoas derecho de $16 \times 2 \mathrm{~cm}$; la punción de esta revela la presencia de flora polimicrobiana, por lo que se deja un nuevo drenaje. Cuarenta y ocho horas más tarde comienza con hipotensión, taquicardia, fiebre, dolor abdominal, hematoquecia y salida de material fecaloideo y hemático por el drenaje. El paciente se estabiliza en la unidad de cuidados intensivos y se realiza una nueva TAC (fig. 2), donde se aprecia una extensa colección retroperitoneal derecha con perforación del colon ascendente y otra pequeña periduodenal. Ante estos hallazgos se decide intervención quirúrgica urgente, en la cual se objetiva un gran plastrón en todo el parietocólico derecho, desde el ciego hasta el ángulo hepático, lleno de heces. La pared posterior del colon derecho está esfacelada y con múltiples perforaciones. En el retroperitoneo hay una gran cavidad llena de esfacelos y coágulos que se extiende hacia el duodeno y hacia la región inguinal. Se realiza hemicolectomía derecha dificultosa con anastomosis ileocólica mecánica laterolateral, desbridamiento y lavado profuso, dejando varios drenajes.

Durante el postoperatorio presenta como complicación una fístula de 2. ${ }^{\mathrm{a}}$ y $3 .^{\mathrm{a}}$ porción duodenal de alto débito, que inicialmente se trata de forma conservadora (con sonda nasoyeyunal durante más de 40 días), pero ante el fracaso de este, se procede a la realización de una gastroscopia, demostrando un gran orificio fistuloso, que se consigue cerrar mediante la colocación de clips endoscópicos, cesando así la mayor parte del débito.

En los días posteriores al cierre de la fístula, se objetiva un aumento inicial del débito coincidiendo con la administración de diuréticos, por lo que se solicita una urografía intravenosa, evidenciándose fístula urinaria en el uréter derecho a nivel proximal, a la altura de las grapas duodenales. Se realiza nefrostomía percutánea.

El paciente, tras 98 días de estancia hospitalaria, se da de alta con resolución de la fístula duodenal y con la nefrostomía, en espera de resolución de lesión ureteral por parte de urología.
El linfangioma es un tumor derivado de un defecto en el desarrollo de los vasos linfáticos ${ }^{2,3}$; histológicamente puede ser capilar, cavernoso o quísticos (macroquístico o microquístico) ${ }^{4}$. Son benignos desde el punto de vista anatomopatológico, pero agresivos en su comportamiento, puesto que pueden invadir estructuras vecinas y tienen una alta tasa de recidiva ${ }^{3}$.

Está presente al nacimiento en el $50 \%$ de los casos, diagnosticándose en un $90 \%$ en los dos primeros años de $v$ vida $^{3}$. Se sitúa en diversas localizaciones a lo largo de todo el organismo, pero fundamentalmente en la axila y el cuello con una frecuencia del 95\%, hallándose en el retroperitoneo en menos del $1 \%^{3-5}$.

Como todo tumor retroperitoneal, presenta una clínica insidiosa e inespecífica, pudiendo aparecer dolor, tumoración, molestias digestivas, síndrome miccional o fiebre de origen desconocido ${ }^{1}$; en ocasiones se pueden complicar con hemorragia, infección, torsión o perforación ${ }^{2,4,5}$.

El diagnóstico se va a realizar mediante pruebas de imagen. La ecografía, la TAC y la resonancia magnética nuclear (RMN) son las técnicas radiológicas complementarias que nos van a ofrecer mayor información ${ }^{2,3,5}$. La ecografía permite diferenciar la naturaleza de la lesión (quiste con paredes delgadas con septos y ecos internos) ${ }^{4,5}$; la TAC y la RMN nos dan información del tamaño, la extensión y las relaciones ${ }^{3,5}$. Hay controversia en la literatura médica acerca de si el diagnóstico de certeza se puede obtener mediante técnicas de imagen o es preciso una confirmación anatomopatológica debido a la aparición de estudios que sugieren un diagnóstico preoperatorio certero con la TAC y la $\mathrm{RMN}^{6,7}$; en cualquier caso, el papel de la punción aspiración con aguja fina es discutible debido a su bajo rendimiento y a sus potenciales complicaciones (diseminación, perforación, etc. $)^{5}$.

En cuanto al tratamiento, la cirugía es el de elección. El problema es que por su carácter agresivo puede encontrarse invadiendo estructuras vecinas vitales, lo que lo haría irresecable ${ }^{5}$; además, una cirugía incompleta conlleva una alta tasa de recurrencia ${ }^{3}$. Por todo esto han surgido diferentes tratamientos alternativos, como radioterapia, diatermia y escleroterapia percutánea ${ }^{1-9}$. En relación con este último, se han empleado distintas sustancias esclerosantes (alcohol, bleomicina, OK-43, etc.), todas ellas con pobres resultados; en los últimos años se está utilizando como esclerosante el ácido acético, sustancia usada en nuestro caso; sin embargo, solo se dispone de series $\operatorname{cortas}^{8}$ y casos aislados ${ }^{9}$ que no ofrecen una clara evidencia científica.

El linfangioma quístico retroperitoneal es una entidad benigna poco frecuente que presenta un carácter invasivo, por lo que requiere una extirpación completa. Actualmente no disponemos de un tratamiento alternativo seguro y eficaz para aquellos casos en los que no es posible una extirpación quirúrgica.

B I B L I O G R A F Í A

1. Blasco Casares FJ. Tumores retroperitoneales. Annals d'Urología. 2003;3.

2. Cruz Guerra NA, Salvador Fernández L, Solera Arroyo JC, Albarrán Fernández M, López Mouriño VM, Alonso Alonso M. Linfangioma quístico retroperitoneal gigante en adulto. Arch Esp Urol. 2005;58:685-8. 
3. Arzoz Fábregas M, Ibarz Servio LL, Areal Calama J, González Satué C, Ruiz Domínguez J, Salidié Roig JM. Linfangioma quístico. Nuestra experiencia. Actas Urol Esp. 2006;30: 723-7.

4. Rani DV, Srilakshmi R, Malathi S, Raghupathy V, Bagdi RK. Unusual presentation of a retroperitoneal lymphangioma. Indian J Pediatr. 2006;73:617-8.

5. Gómez Pascual JA, Martin Morales A, Bonilla Parrilla R, Alvarado Rodríguez A, Blanco Reina F, Rodero García P, et al. Linfangioma quístico retroperitoneal. Una patología silente en adultos. Actas Urol Esp. 2002;28:356-60.

6. Bonhomme A, Broeders R, Oyen RH, Stas M, De Wever I, Baert AL. Cystic lymphangioma of the retroperitoneum. Clin Radiol. 2001;56:156-8.

7. Wunderbaldinger P, Paya K, Partl B, Turetschek K, Hormann M, Horcher E, et al. CT and MR imaging of generalized cystic lymphangiomatosis in pediatric patients. AJR Am J Roentgenol. 2000;174:827-32.
8. Hwan Won JE, Moon Kim B, Kim C, Woo Park S, Deuk Kim M. Percutaneous sclerotherapy of lymphangiomas with acetic acid. J Vasc Inter Radiol. 2004;15:595-600.

9. Woo Park S, Ho Cha I, Ah Kim K, Joo Hong S, Min Park C, Hoon Chung H. Percutaneous sclerotherapy using acetic acid after failure of alcohol ablation in an intra-abdominal lymphangioma. Cardiovasc Intervent Radiol. 2004;27:285-7.

M. Mengual-Ballester*, R. Montoya-Chinchilla, M.P. Guillén-Paredes y J.L. Aguayo-Albasini

Servicio de Cirugía General, Hospital General Universitario JM Morales Meseguer, Murcia, España

*Autor para correspondencia.

Correo electrónico: mmengual@msn.com

(M. Mengual-Ballester).

\section{Cistadenoma prostático (quiste multilocular de la próstata) Cystadenoma of the prostate (multilocular cyst of the prostate)}

\section{Sr. Director:}

Presentamos el caso de un paciente varón de 40 años que consultó por sensación de retención de orina; en la ecografia se halló una lesión quística de la línea media de la próstata, con crecimiento endovesical, causando residuos posmiccionales altos (fig. 1). En una urografía endovenosa se pudo comprobar que no se trataba de un ureterocele ectópico. Los análisis de rutina y la determinación de PSA fueron normales. Bajo anestesia, se realizó resección endoscópica de una lesión con múltiples quistes de tamaño variable, generalmente

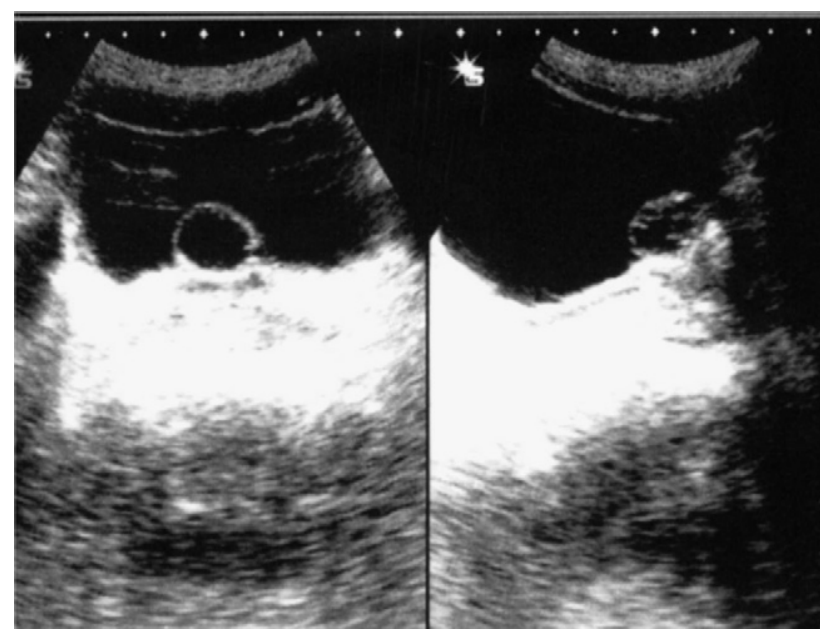

Figura 1 - Ecografía hipogástrica que muestra lesión quística en la línea media prostática, de crecimiento endovesical. pequeños, situada en el hemicontorno superior de la uretra prostática y en parte del hemitrígono izquierdo.

En el estudio anatomopatológico se encontró una proliferación quística multilocular, tapizada por una o más capas de epitelio cilíndrico o cuboideo, sin atipia (fig. 2). El estudio inmunohistoquímico con anticuerpos anti-PSA fue positivo en las células que revestían las cavidades quísticas. Tres años después, permanece sin síntomas miccionales ni signos de recidiva.

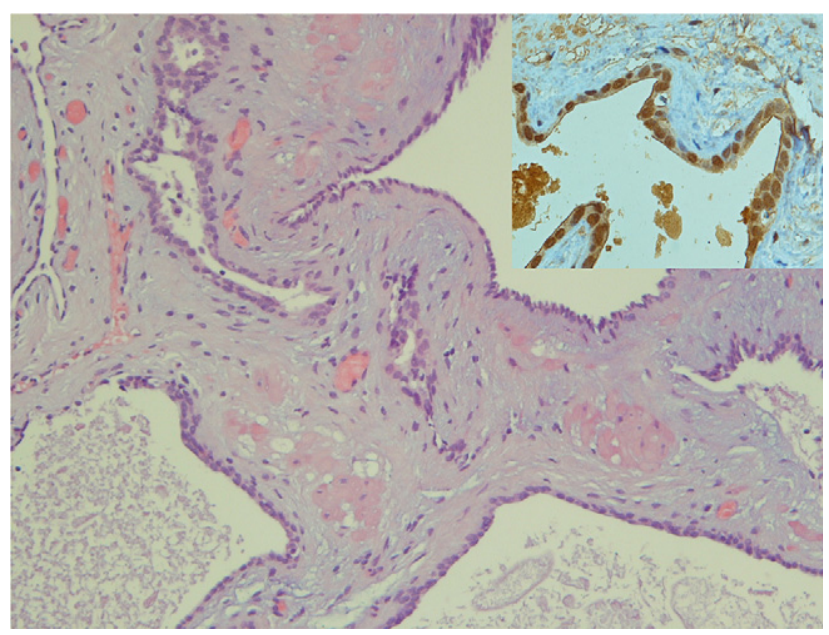

Figura 2 - Lesión quística multiloculada, revestida por epitelio cilíndrico aplanado. Imagen insertada (arriba a la derecha): se muestra que la tinción inmunohistoquímica para PSA es positiva. 